\title{
INTRODUCTION TO THE SPECIAL ISSUE ON MODELING AND SIMULATION APPLICATIONS OF SOFTWARE AGENTS
}

\section{Dear SCPE readers,}

The aim of this Special Issue is to introduce to the readers a selection of papers from the 3rd Workshop on Applications of Software Agents WASA 2013 in the area of agent-based modelling and simulation. WASA2013 was held in Sinaia, Romania, during October 11-13, 2013. The WASA 2013 workshop was organized within the framework of the 17th International Conference on System Theory, Control and Computing ICSTCC2013. The aim of the WASA series of workshops is to contribute to the advancement of technologies and applications of software agents' by bridging the gap between the theory and practice of software agents.

15 papers were accepted for presentation at WASA2013. Among them, 3 papers were selected, after further extension and additional review, for inclusion in this Special Issue on Modeling and Simulation Applications of Software Agents.

The article Formal Modelling and Simulation of a Multi-Agent Nano-Robotic Drug Delivery System by Marina Ntika, Petros Kefalas, and Ioanna Stamatopoulou is in the area of agent-based modelling and simulation. The authors propose an agent-based simulation model for targeted drug delivery with the help of nano-robots. The simulation model is developed using X-machines, while the simulation itself was achieved using NetLogo. The paper reports interesting experimental results that were obtain by applying the proposed agent-based simulation model, thus supporting the usefulness of formal multi-agent system for the better understanding of collaborative nano-robotic technologies for drug delivery in unhealthy tissues.

The article A System Supporting the Evaluation of the Operational Effectiveness of Naval Tasks Based on Agent Simulation by Davide Anghinolfi, Alberto Capogrosso, Massimo Paolucci, and Perra Francesco is in the area of agent-based modelling and simulation. The authors propose an agent-based simulation model denoted as denoted as Operational Evaluator Model, for the multi-dimensional analysis of alternative configurations of military naval units to support the design of naval vessels. After the careful consideration of several existing frameworks for agent-based simulation, the authors propose their own framework denoted as ABSF. The paper contains convincing experimental results obtained by simulation of a specific Anti-surface Warfare use case that support the advantages of ABSF over other agent-based simulation frameworks.

The article ABVE-Construct: An Agent-Based Virtual Enterprise Model for Civil Engineering by Mihaela Oprea is in the area of agent-based enterprise modelling. This paper proposes a generic framework for virtual enterprises development denoted as VE-Frame. Based on this framework, a new agent-based virtual enterprise model for the civil engineering domain denoted as ABVE-Construct, is formulated. The ABVE-Construct ontology and design model are developed using OWL and Protg, as well as using Prometheus Design Tool. The proposed framework and model are evaluated by considering a specific case study of a residential building construction task.

We would also like to thank all the reviewers for their restless reviewing effort and valuable feedback and all the authors who submitted papers to WASA2013 and to this Special Issue.

Costin Bădică and Mirjana Ivanović 